\title{
Associations between neurofilament light chain levels, disease activity and brain atrophy in progressive multiple sclerosis
}

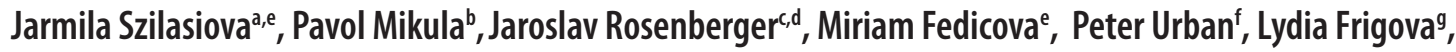 \\ Marianna Vitkovaa,e, Zuzana Gdovinova ${ }^{\mathrm{a}, \mathrm{e}}$, Jozef Hanes ${ }^{\mathrm{h}}$, , Eva Stevens ${ }^{\mathrm{i}}$
}

Background. Neurofilament light chain is a promising biomarker of disease activity and treatment response in relapsing-remitting multiple sclerosis (MS). Its role in progressive MS is less clear.

Aim: The aim of the study was to assess the relationship between plasma neurofilament light chain ( $\mathrm{pNfL}$ ) and disease activity as defined by the concept NEDA-3 (No Evident Disease Activity), and brain volumetry, in a cohort of patients with the progressive disease form (PMS).

Methods. Levels of pNfL (SIMOA technology) were examined in 52 PMS patients and analysed in relationship to NEDA-3 status and annual brain volume loss (BVL) during the last 12 months. The statistical model was developed using logistic regression analysis, including demographic, clinical and magnetic resonance imaging (MRI) data as independent variables. Dependent variables were NEDA-3 status and BVL.

Results. The mean age of the study participants ( $n=52,50 \%$ females) was 45.85 (SD, 9.82) and the median disability score was 5.0 (IQR: 5.0-5.5). ROC analysis showed that pNfL predicts NEDA-3 (the sensitivity and specificity of the model were $77.8 \%$ and $87.6 \%$, respectively, $P<0.001$ ) and abnormal BVL (the sensitivity and specificity were $96.6 \%$ and $68.2 \%$, respectively, $P<0.001$ ).

Conclusions. The results show that pNfL levels are a useful biomarker of disease activity determined by NEDA-3 status, including brain MRI-volumetry, in patients with the progressive form of MS.

Key words: multiple sclerosis, progressive MS, neurofilament light chain, no evident disease activity, brain volume loss

Received: January 25, 2021; Revised: April 13, 2021; Accepted: May 24, 2021; Available online: June 1, 2021

https://doi.org/10.5507/bp.2021.034

(c) 2022 The Authors; https://creativecommons.org/licenses/by/4.0/

${ }^{a}$ Department of Neurology, Pavol Jozef Safarik University in Kosice, Slovak Republic

${ }^{b}$ Department of Social and Behavioral Medicine, Pavol Jozef Safarik University in Kosice, Slovak Republic

'Department of Health Psychology and Methodology of Research, II. Internal Clinic, Pavol Jozef Safarik University in Kosice, Slovak Republic ${ }^{d}$ Olomouc University Social Health Institute, Palacky University Olomouc, Czech Republic

${ }^{e}$ Department of Neurology, L. Pasteur University Hospital, Kosice, Slovak Republic

${ }^{f}$ Department of Medical and Clinical Biochemistry, Faculty of Medicine, Pavol Jozef Safarik University in Kosice, Slovak Republic

${ }^{g}$ Pro Magnet Kosice, Slovak Republic

hInstitute of Neuroimmunology, Slovak Academy of Sciences, Bratislava, Slovak Republic

'AXON Neuroscience R\&D Services SE, Bratislava, Slovak Republic

Corresponding author: Jarmila Szilasiova, e-mail:jarmila.szilasiova@upjs.sk

\section{INTRODUCTION}

Current research in multiple sclerosis (MS) is focused on the identification of sensitive biomarkers related to disease activity in all MS phenotypes ${ }^{1-6}$. In MS patients treated with DMTs (disease modifying therapy) treatment response is measured using NEDA (No Evident Disease Activity) status ${ }^{7}$. Three-compound NEDA-3 status takes account of the absence of relapse, brain magnetic resonance imaging (MRI) activity and worsening disability during the last year. Some researchers point out that NEDA-3 status mostly reflects inflammatory activity and overlooks ongoing neurodegenerative processes.

In progressive MS (PMS) forms, which include secondary progressive MS (SPMS) and primary progressive MS (PPMS), treatment effect is evaluated using NEDA-3 status despite the fact that inflammatory activity is low- er. Following the NEDA-3 concept may not capture the whole disease activity in PMS, in that PMS is characterised by a steady accumulation of disability independent of relapses ${ }^{8}$.

One proposal to address these limitations is the measurement of brain volume loss (BVL), a marker of neurodegeneration. Clinical drug trials that involved BVL as an outcome parameter have shown that the effect of treatment on BVL correlated with the effect on disability progression ${ }^{9-11}$.

Neurofilament light chain (NfL), cytoskeletal proteins confined to the neuroaxonal compartment, has been identified as a promising biomarker in MS related to neuronal damage, disease activity and treatment response $\mathrm{e}^{1-6,9-13}$. Brain volume loss, determined as a mean annual BVL rate (AR-BVL) of $<0.4 \%$, was identified as the annual threshold BVL rate in MS patients in several studies ${ }^{12,1416}$. 
Sormani et al. concluded that NfL could possibly be used as a substitute marker for brain volume loss ${ }^{11}$.

We investigated in a PMS (SPMS and PPMS) cohort plasma NfL ( $\mathrm{pNfL}$ ) levels, parameters of disease activity as defined by NEDA-3 and brain volumetry, including whole brain volume, grey matter volume and BVL. We then analysed the relationship between pNfL and demographic, clinical and radiological parameters, as well as associations with NEDA-3 and BVL in our PMS cohort. To the best of our knowledge these variables have not been studied in such a context.

\section{METHODS}

\section{Study Population}

Fifty-two consecutive patients with PMS ( 5 with PMS and 47 with SPMS) were eligible to participate in the study and enrolled in a cohort study at the Department of Neurology of Louis Pasteur University Hospital in Košice (also see Fig. 1 for the study design). The study was approved by the Louis Pasteur University Hospital Ethics Committee (2017/EK/4005) and was performed in accordance with the Good Clinical Practice standard and the Declaration of Helsinki.

The inclusion criteria were the following: (1) diagnosis of primary progressive MS or secondary progressive MS in accordance with the phenotypic MS classification ${ }^{8}$, (2) being older than 18 years, and (3) the ability to give written informed consent. The exclusion criterion was a severe comorbidity. Of the patient population involved in the study $(n=52), 23$ were males and 29 were females. All patients were treated with DMTs according to Slovak MS treatment criteria. The study period was January 2019 February 2020.

Patients underwent clinical neurological examination, including expanded disability status scale (EDSS) ( ref. $^{17}$ ), plasma NfL ( $p$ NfL) sampling and brain MRI. Patient characteristics are presented in Table 1. Disease duration was considered the time from the first symptoms of MS to the date of the pNfL examination.

\section{Study design}

The baseline visit was 12 months before the followup visit. The baseline visit included compliance with the inclusion criteria (see above), demographic data (age, gender), clinical data (disease duration, EDSS score), MRI with MRI volumetry and current DMT. The followup visit included a pNfL sample, history in the last 12 months relapse, EDSS score and EDSS change in the last 12 months, MRI with MRI volumetry and current DMT. EDA (Evident disease activity) status was unfilled NEDA-3 status.

\section{NfL measurements}

The NfL sample was taken at the follow-up visit (12 months after the baseline visit). Samples of $3 \mathrm{ml}$ of venous blood were collected into vacutainer tubes including an anticoagulant (sodium citrate) by the treating physicians and processed at room temperature within two hours. The samples were then spun at $4000 \mathrm{rpm}$ for 10 minutes, and the collected plasma was divided into polypropylene tubes and stored at $-80{ }^{\circ} \mathrm{C}$. The blood samples were pseudonymized and analysed without clinical data.

The pNfL were analysed by SIMOA $^{\mathrm{TM}}$ (Single Molecule Array) NfL assay using the NF-Light Advantage Kit and the SIMOA HD-1 analyser, protocol of Quanterix, Lexington, MA, USA (ref. ${ }^{18}$ ). The inter-assay coefficients of variation for three native serum samples were $5.3 \%, 1.9 \%$ and $5.3 \%$ for $6.2,21.7$ and $256 \mathrm{pg} / \mathrm{mL}$, respectively. The intra-assay coefficients of variation $(n=30)$ for three native serum samples were $6.1 \%, 6.8 \%$ and $7.3 \%$ for $6.2,21.7$ and $256 \mathrm{pg} / \mathrm{mL}$, respectively.

\section{MRI measurements}

Brain MRI was performed using a standardized threedimensional (3D) T1-weighted magnetization-prepared rapid gradient-echo sequence and a 3D T2-weighted fluid-attenuated inversion recovery (FLAIR) sequence with MS protocol in all PMS patients. Lesion maps were initially drawn on T2-weighted 3D FLAIR images using a PHILIPS Ingenia 3.0T Omega HP (Philips North America Corporation, dStream, Direct digital technology). Longitudinal coregistration fusion was used for identification of T2 lesions, FLAIR lesions, T1 lesions, T1 gadolinium-enhancing lesions and their occurrence,

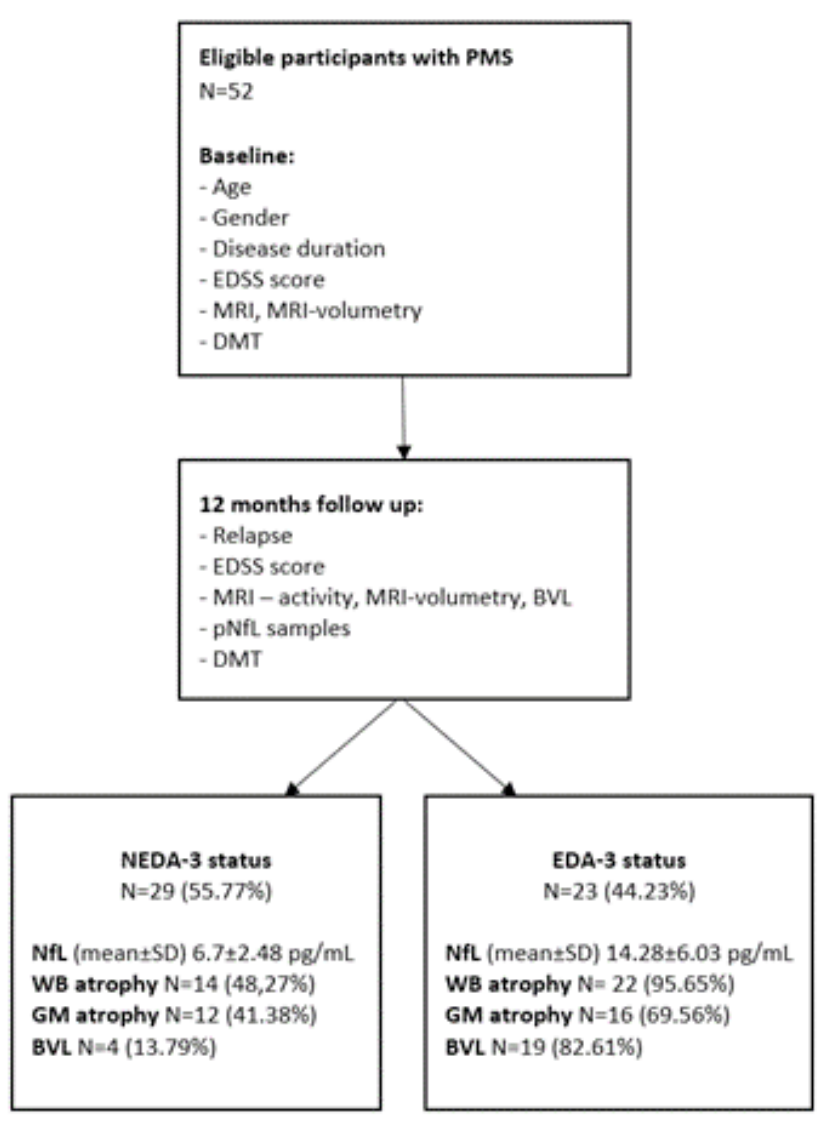

Fig. 1. A schematic flowchart diagram showing the procedure by which data were tested in MS patients.

EDA, Evident disease activity; NEDA, No evident disease activity; BVL, Brain volume loss; MS, Multiple sclerosis. 
as well as new or enlarged lesions, and their volume was measured.

Whole brain (WB) volume and grey matter (GM) volume were measured using the Icobrain program (ICOMETRIX). Volumetric parameters were calculated by automatic brain volume quantification using FLAIR and T1-weighted scans using longitudinal, trans-sectional and segmentation techniques ${ }^{19}$. The Icobrain program compares the measured values of brain volumes in patients with those of healthy controls and calculates the deviation from the standard values evaluated in healthy controls (the database is mainly from Europe and North America), with the average expected annual change in volume for controls that match in the age and gender category.

Whole brain volume and grey matter volume parameters were adjusted for skull size using Icobrain, and the normal range and normative volume percentile change in healthy controls was used as a reference.

WB atrophy was determined as abnormal annual WB volume change. This parameter is based on the annual percentage of whole brain volume change as a value above the normal range of reference values in healthy controls (database), in accordance with gender and age (Icobrain program). GM atrophy was determined as abnormal annual GM volume change based on the annual percentage grey matter volume change with value above the normal range of reference values in healthy controls (database), in accordance with gender and age (Icobrain program).

BVL (brain volume loss) was defined as an annual BVL rate (AR-BVL) above $0.4 \%$ according to the study of De Stefano et al. ${ }^{14}$. Patients were dichotomized based on an AR-BVL $<0.4 \%$ or $\geq 0.4 \%$ (BVL).

Brain MRI was performed within the last 3 months before the baseline visit and the follow-up visit (Fig.1). MRI data were analysed by blinded radiologists and Icobrain raters, who had no information about disease activity and NfL levels.

\section{NEDA-3 definition}

NEDA-3 status was evaluated using data from the last 12 months. NEDA-3 status was defined as the absence of relapse, EDSS worsening and MRI activity.

EDSS worsening was defined as an increase in the EDSS score of 1.5 points, if the previous EDSS score was (a baseline score) 0 ; an increase of $\geq 1.0$ point, if EDSS $\leq 5.0$; or an increase of $\geq 0.5$ points, if EDSS $\geq 5.5$, confirmed at 6 months ${ }^{20,21}$.

MRI activity was defined as having at least two or more new/or enlarging T2-hyperintense lesions or the presence of a gadolinium (Gd)-enhancing T1 lesion in the brain MRI (at follow-up visit) compared with the previous scan (baseline visit).

\section{Statistical analysis}

Descriptive statistics were compiled to provide basic information about the patients. Summary statistics are presented as the mean $\pm \mathrm{SD}$, median (range) and percentage, where applicable categorical variables are number and percentage. Thereafter, binary logistic regression analysis was performed. The model included demographic (age, gender), laboratory ( $\mathrm{pNfL}$ ), clinical (EDSS, disease duration) and MRI variables as independent variables. Dichotomized WB atrophy, GM atrophy, NEDA-3 status and BVL status were used as the dependent variables. The conditional backward stepwise method was used to select the model with the best predictors. Consequently, for evaluation of the predictive value of $\mathrm{pNfL}$ levels as a predictor of WB atrophy, GM atrophy, NEDA-3 status and BVL status, we plotted the ROC (Receiver operating characteristics) curve and calculated the area under curve (AUC) with a 95\% confidence interval (CI). Optimal NfL cut-offs were defined based on the Youden index. From the pNfL cut-off values, we selected the optimal one with the highest discriminant accuracy (sensitivity and specificity).

Statistical analyses were performed at the 0.05 level/ values of significance using the IBM SPSS (Statistical Package for the Social Science) software version 23.0.

\section{RESULTS}

The demographic, clinical, laboratory and radiological (MRI) characteristics of the patients with PMS are provided in Table 1 . The mean age was $45.87 \pm 9.82$ years, and $29(55.77 \%)$ patients were female. The median disease duration was 16.0 (IQR: 9.25-21.75) years, and the median pNfL was $11.1 \mathrm{pg} / \mathrm{mL}$ (IQR: 5.2-11.13). Patients were treated with DMTs in the following proportions: 22 patients $(42.31 \%)$ were on first line DMTs and 30 $(57.69 \%)$ on second line DMTs (Table 1$)$.

From a total of $52(100 \%)$ patients, 29 (55.77\%) had NEDA-3 status in the past evaluated year, while $23(44.23 \%)$ patients showed EDA-3 status. The pNfL were significantly higher in the EDA-3 group than in the NEDA-3 group $(14.28 \pm 6.03 \mathrm{pg} / \mathrm{mL}$ vs $6.7 \pm 2.48 \mathrm{pg} /$ $\mathrm{ml} ; P<0.001)$.

Thirty-six $(69.23 \%)$ patients met the criteria for WB atrophy and $28(53.85 \%)$ patients met the criteria for GM atrophy. Twenty-three (44.23\%) patients met the criteria for BVL (AR-BVL $\geq 0.4 \%$ ); 4 of them had NEDA-3 status, and 19 had EDA-3 status (Fig. 1.).

From the subgroup of patients with NEDA-3 status $(n=29), 4(13.79 \%)$ patients had BVL, 12 (41.38\%) had GM atrophy and 14 (48.27\%) had WB atrophy.

From the subgroup of patients with EDA-3 status $(n=23), 19(82.61 \%)$ patients had BVL, 16 (69.56\%) had GM atrophy, and 22 (95.65\%) had WB atrophy (Fig. 1).

Multivariable logistic regression analysis, a model consisting of age, gender, disease duration, EDSS (at follow-up visit) and $\mathrm{pNfL}$ as independent variables and NEDA-3 status as the dependent variable, showed that the $\mathrm{pNfL}$ value $(\mathrm{B}=-0.563, \operatorname{Exp}(\mathrm{B}) 0.57 ; P<0.001)$ is a significant predictor of NEDA-3 status. ROC curve analysis showed the pNfL to be a predictor of NEDA-3 status (AUC $=0.848 ; 95 \%$ CI:0.739-0.957; $P<0.001$ ), and the sensitivity and specificity of the predictive model were $77.8 \%$ 
Table 1. Demographic, clinical, laboratory and MRI characteristics of the study sample.

\begin{tabular}{|c|c|}
\hline \multicolumn{2}{|l|}{ Demographic characteristics } \\
\hline Sample size, $\mathrm{n}$ & 52 \\
\hline Female, n (\%) & $29(55.77)$ \\
\hline Age (years), mean (SD) & $45.87(9.82)$ \\
\hline \multicolumn{2}{|l|}{ Clinical characteristics } \\
\hline Disease duration (years), median, IQR & $16(9.25-21.75)$ \\
\hline EDSS, median, IQR & $5.0(5.0-5.5)$ \\
\hline Proportion of patients with last year relapse, $\mathrm{n}(\%)$ & $20(38.46)$ \\
\hline Proportion of patients with EDSS worsening, n (\%) & $18(34.62)$ \\
\hline \multicolumn{2}{|l|}{ Laboratory characteristics } \\
\hline pNfL levels, pg/mL, median, IQR & $11.1(5.2-11.13)$ \\
\hline \multicolumn{2}{|l|}{ MRI measures } \\
\hline WB volume, median, IQR & $1461(1403.25-1461)$ \\
\hline WB volume normative percentile, median, IQR & $0.98(0.8-6.7)$ \\
\hline WB annual volume change, median, IQR & $-0.52(-0.79--0.23)$ \\
\hline GM volume, median, IQR & $858.5(826-888)$ \\
\hline GM volume normative percentile, median, IQR & $2.6(0.9-15.5)$ \\
\hline GM annual volume change, median, IQR & $-0.4(-0.71--0.21)$ \\
\hline Proportion of patients with MRI activity, n (\%) & $14(26.92)$ \\
\hline Proportion of patients with BVL, n (\%) & $23(44.23)$ \\
\hline Proportion of patients with WB atrophy, n (\%) & $36(69.23)$ \\
\hline Proportion of patients with GM atrophy, n (\%) & $28(53.85)$ \\
\hline \multicolumn{2}{|l|}{ NEDA-3 status } \\
\hline Proportion of patients with NEDA-3, n (\%) & $29(55.77)$ \\
\hline Proportion of patients with EDA-3, n (\%) & $23(44.23)$ \\
\hline \multicolumn{2}{|l|}{ Therapy } \\
\hline Proportion of patients with first line DMT, $\mathrm{n}(\%)$ & $22(42.31)$ \\
\hline Proportion of patients with interferon-beta $\mathrm{n}(\%)$ & $4(7.69)$ \\
\hline Proportion of patients with teriflunomide, $\mathrm{n}(\%)$ & $6(11.54)$ \\
\hline Proportion of patients with dimethylfumarate, n (\%) & $12(23.08)$ \\
\hline Proportion of patients on second line DMT, $\mathrm{n}(\%)$ & $30(57.69)$ \\
\hline Proportion of patients with fingolimod n (\%) & $7(13.46)$ \\
\hline Proportion of patients with ocrelizumab, n (\%) & $7(13.46)$ \\
\hline Proportion of patients with cladribine, n (\%) & $10(19.23)$ \\
\hline Proportion of patients with alemtuzumab, n (\%) & $6(11.54)$ \\
\hline
\end{tabular}

Continuous data are calculated with mean $\pm \mathrm{SD}$ (standard deviation); $\mathrm{pNfL}$ are median (IQR); categorical variables are number (\%).

Legend: AR-BVL, Annual Rate Brain Volume Loss; BVL, Brain Volume Loss; DMT, Disease Modyfying Therapy; EDSS, Expanded Disability Status Scale; IQR, Interquartile Range; MRI, Magnetic Resonance Imaging; NEDA, No Evident Disease Activity; pNfL, Plasma Neurofilament Light Chain; WB, Whole Brain; GM, Grey Matter

and $87.57 \%$, respectively (Fig. 2). Lower pNfL values indicate stronger evidence for the presence of NEDA-3 status, with a cut-off level of $9.1 \mathrm{pg} / \mathrm{mL}$ for $\mathrm{pNfL}$.

Multivariable logistic regression analysis, a model consisting of age, gender, disease duration, EDSS and pNfL as independent variables and WB atrophy as the dependent variable, showed that the $\mathrm{pNfL}$ value $(\mathrm{B}=0.528$, $\operatorname{Exp}(\mathrm{B}) 1.695 ; P<0.05)$ is a significant predictor of $\mathrm{WB}$ atrophy. ROC curve analysis showed the $\mathrm{pNfL}$ value to be a predictor of $\mathrm{WB}$ atrophy $(\mathrm{AUC}=0.905 ; 95 \% \mathrm{CI}$ : $0.803-1.0 ; P<0.001)$, and the sensitivity and specificity of the predictive model were $93.5 \%$ and $75 \%$, respectively (Fig. 3). Higher pNfL values indicate stronger evidence for the presence of WB atrophy, with a cut-off level of 8.5 $\mathrm{pg} / \mathrm{mL}$ for $\mathrm{pNfL}$.
Multivariable logistic regression analysis, a model consisting of age, gender, disease duration, EDSS and pNfL as independent variables and GM atrophy as the dependent variable, showed that the $\mathrm{pNfL}$ value $(\mathrm{B}=0.129$, $\operatorname{Exp}(\mathrm{B}) 1.137 ; P=0.097$ ) is a predictor of $\mathrm{GM}$ atrophy but did not show a level of statistical significance. ROC curve analysis showed the $\mathrm{pNfL}$ value to be a predictor of GM atrophy (AUC=0.738; 95\% CI: 0.587-0.809; $P<0.01)$, and the sensitivity and specificity of the predictive model were $71.9 \%$ and $73.7 \%$, respectively (Fig. 4). Higher pNfL values indicate stronger evidence for the presence of GM atrophy, with a cut-off level of $9.55 \mathrm{pg} / \mathrm{mL}$ for $\mathrm{pNfL}$.

Multivariable logistic regression analysis, a model consisting of age, gender, disease duration, EDSS and pNfL as independent variables and BVL as the dependent 


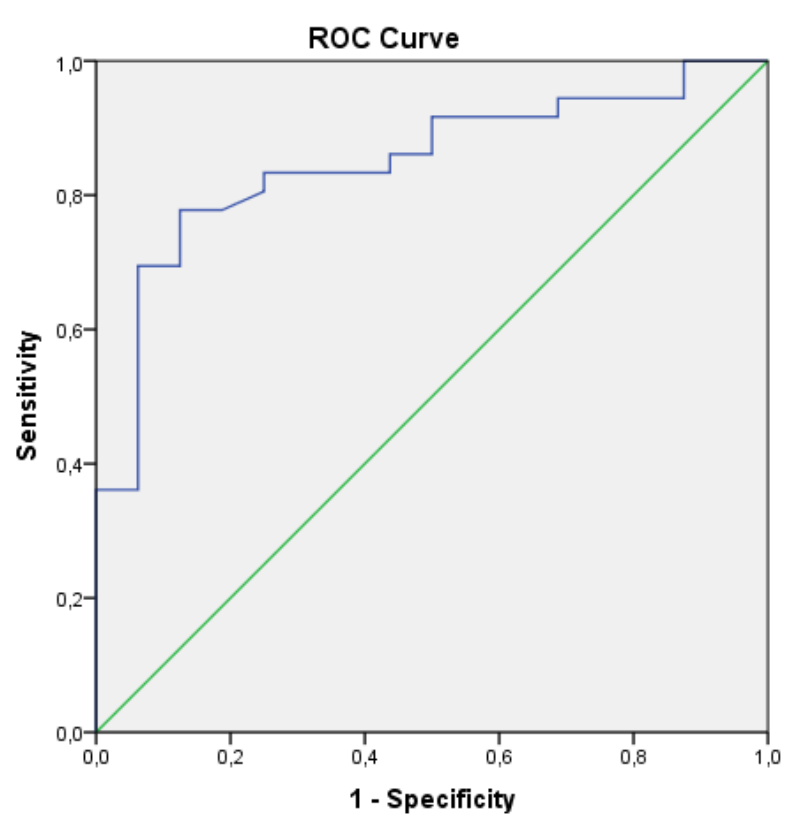

Diagonal segments are produced by ties.

Fig. 2. ROC analysis of the pNfL of patients with and without NEDA-3 status. The sensitivity and specificity of the predictive model were $77.8 \%$ and $87.5 \%$, respectively, with a cut-off level of $9.1 \mathrm{pg} / \mathrm{mL}$ for $\mathrm{pNfL}$, and an area under curve (AUC) of 0.848 (95\% CI: $0.739-0.957 ; P<0.001$ ). Lower $\mathrm{pNfL}$ values indicate stronger evidence for the presence of NEDA-3 status.

AUC, Area under curve; NEDA, No evident disease activity; pNfL, plasma neurofilament light chain; ROC, Receiver operating characteristics.

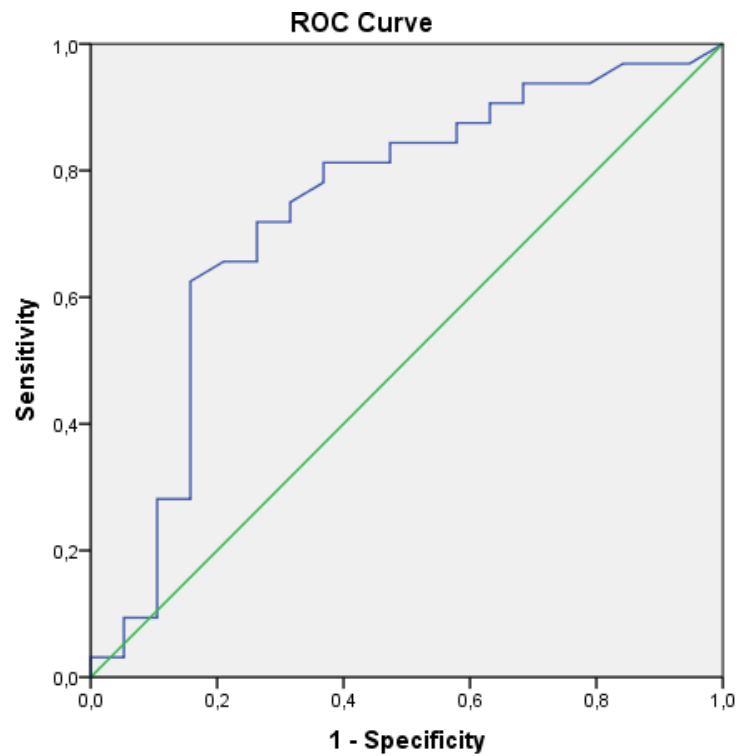

Diagonal segments are produced by ties.

Fig. 4. ROC analysis of the pNfL of patients with and without GM atrophy. The sensitivity and specificity of the predictive model were $71.9 \%$ and $73.7 \%$, respectively, with a cut-off level of $9.55 \mathrm{pg} / \mathrm{mL}$ for NfL, and an area under curve (AUC) of 0.738 (95\% CI: 0.587-0.809; $P<0.01$ ). Higher $\mathrm{pNfL}$ values indicate stronger evidence for the presence of GM atrophy.

AUC, Area under the curve; GM, Gray matter; NEDA, No evident disease activity; pNfL, plasma neurofilament light chain; ROC, Receiver operating characteristics.

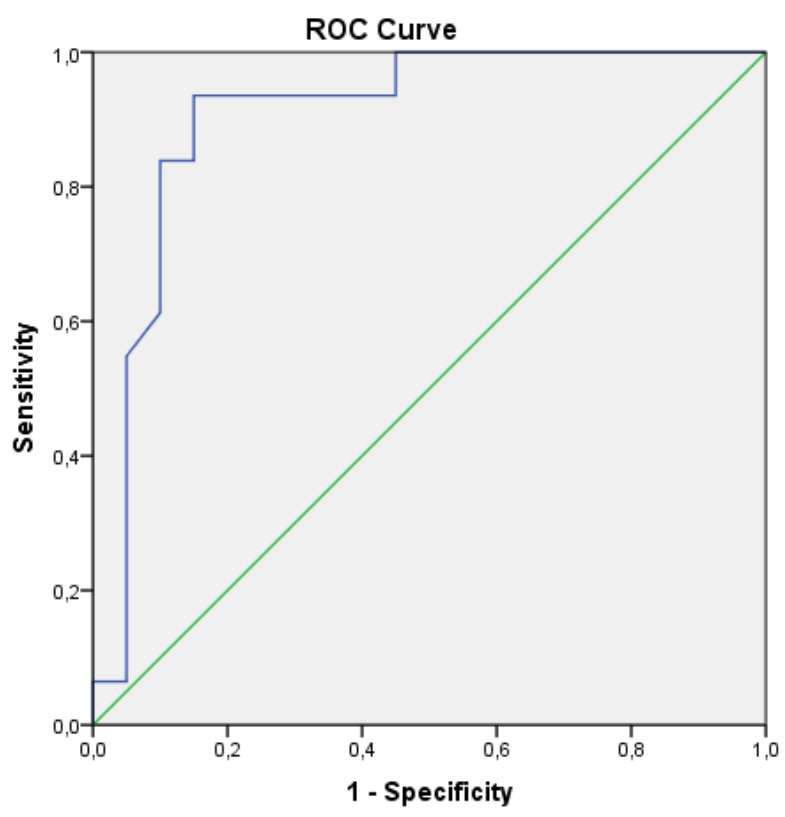

Diagonal segments are produced by ties.

Fig. 3. ROC analysis of the pNfL of patients with and without WB atrophy. The sensitivity and specificity of the predictive model were $93.5 \%$ and $75 \%$, respectively, with a cut-off level of $8.5 \mathrm{pg} / \mathrm{mL}$ for NfL, and an area under curve (AUC) of 0.905 (95\% CI: $0.803-1.0 ; P<0.001)$. Higher $\mathrm{pNfL}$ values indicate stronger evidence for the presence of WB atrophy.

AUC, Area under the curve; NEDA, No evident disease activity; pNfL, plasma neurofilament light chain; ROC, Receiver operating characteristics; WB, Whole brain.

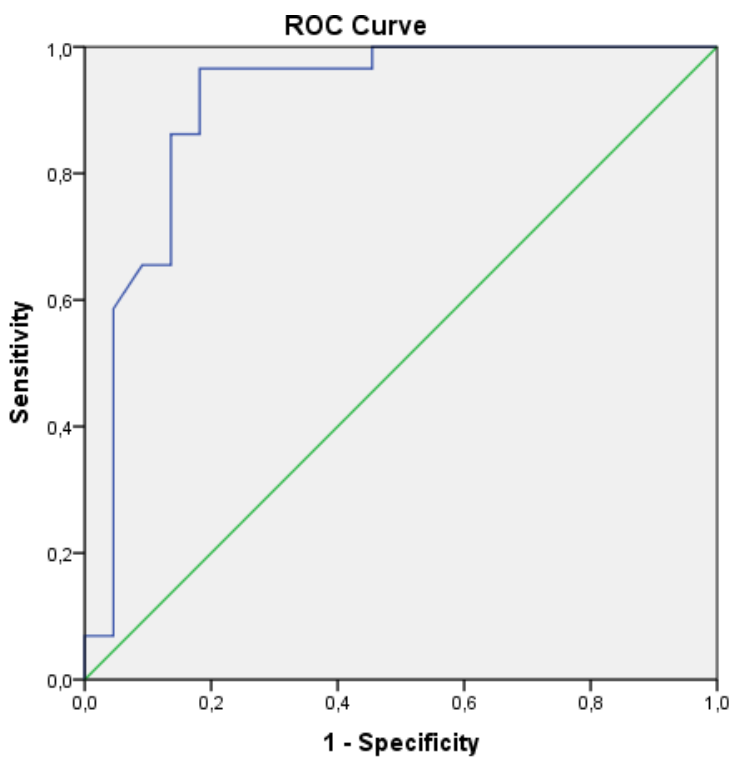

Diagonal segments are produced by ties.

Fig. 5. ROC analysis of the pNfL of patients with and without BVL. The sensitivity and specificity of the predictive model were $96.6 \%$ and $68.2 \%$, respectively, with a cut-off level of 8.25 $\mathrm{pg} / \mathrm{ml}$ for NfL, and an area under curve (AUC) of 0.909 (95\% CI: $0.815-1.0 ; P<0.001)$. Higher $\mathrm{pNfL}$ values indicate stronger evidence for the presence of BVL.

AUC, Area under the curve; BVL, Brain volume loss, NEDANo evident disease activity; pNfL, plasma neurofilament light chain; ROC, Receiver operating characteristics. 
variable, showed that the $\mathrm{pNfL}$ value $(\mathrm{B}=0.564, \operatorname{Exp}(\mathrm{B})$ $1.758 ; P<0.001)$ is a significant predictor of BVL. ROC curve analysis showed the pNfL level to be a predictor of BVL (AUC $=0.909 ; 95 \% \mathrm{CI}=0.815-1.0 ; P<0.001)$, and the sensitivity and specificity of the predictive model were 96.6\% and 68.2\%, respectively (Fig. 5). Higher pNfL values indicate stronger evidence for the presence of BVL, with a cut-off level of $8.25 \mathrm{pg} / \mathrm{mL}$ for $\mathrm{pNfL}$.

\section{DISCUSSION}

The results of our study showed that plasma neurofilament light chain levels are predictors of disease activity in progressive MS forms, as measured by 3-domain NEDA3 status, consisting of the absence of relapse, disability (EDSS) worsening and brain MRI activity. The second main result is that the $\mathrm{pNfL}$ level predicts brain volume parameters, specifically whole brain atrophy, gray matter atrophy and brain volume loss $(\geq 0.4 \%)$. From a total of 52 patients with PMS on DMT treatment, more than half (55.8\%) had NEDA-3 status, and $86.2 \%$ of them were without BVL. More than half (55.8\%) of all the patients with PMS in the sample were without BVL, and $44.2 \%$ met the criteria for BVL.

The results of ROC curve analyses showed that $\mathrm{pNfL}$ predicts BVL with higher sensitivity $(96.6 \%)$ when compared to WB atrophy (93.5\%), GM atrophy (71.9\%) and NEDA-3 (77.8\%). This may also be reflected by the AUC values: BVL-AUC (0.909) was higher than WB atrophyAUC (0.905), GM atrophy-AUC (0.738) and NEDA-3AUC (0.848). Our results show good potential for $\mathrm{pNfL}$ levels to discriminate between patients with and without NEDA-3 status and the good performance of $\mathrm{pNfL}$ levels in discriminating between patients with and without abnormal BVL.

A comparison of pNfL levels in the NEDA-3 and EDA-3 subgroups showed significant higher levels in the EDA-3 group $(6.7 \pm 2.48$ vs $14.28 \pm 6.03)$. These results are consistent with the findings of several studies s,22-25 $^{5}$ and they seem to indicate that $\mathrm{pNfL}$ reflects disease activity measured by 3-domain NEDA-3 status. In addition, it may support ability of immunosuppressive DMTs to supress NfL.

In the NEDA-3 group $13.8 \%$ of patients had BVL, while in EDA-3 group BVL was present in $82.6 \%$. This shows that BVL probably well captures the disease activity in PMS; similar results have been presented in a few studies with progressive MS (ref. ${ }^{24-27}$ ). In fact, this allows us to conclude that an analysis with $\mathrm{pNfL}$ as the dependent variable may better quantify the association between $\mathrm{pNfL}$ and recent BVL and indicate whether $\mathrm{pNfL}$ and BVL may be somehow complementary. Based on this observation, BVL, along with pNfL, appears to be a useful tool in evaluating progressive MS.

Progressive multiple sclerosis (PMS) is characterised by a steady accumulation of disability largely independent of relapses ${ }^{8}$. Progression in both SPMS and PPMS may occur either in association with inflammatory activity or in the absence of such inflammatory activity ${ }^{8}$; therefore, there is a need to determine sensitive biomarkers pointing to disease activity particularly in "non-active" PMS.

NEDA-3 status is a widely used target of DMT in MS patients in clinical studies as well as in routine practice. Three-domain NEDA status is considered annually in a patient before a decision is made regarding the continuing or switching of DMT therapy. One of the known disadvantages of NEDA-3 is that it mainly reflects the inflammatory component, while neurodegenerative processes can progress undetected; additionaly, the EDSS scale (used for disabilty evaluation) may not accurately detect the progression of disability. It is known that disability progression in patients, despite their meeting the criteria of NEDA-3, is possibly a consequence of brain and spinal cord atrophy ${ }^{27}$. Thus, we chose BVL status for analysis of the relationship between $\mathrm{pNfL}$ and disease activity.

In contrast to other studies in patients with early MS and RRMS, we studied a cohort of patients with PMS with ongoing disease who were on DMT treatment, and our results show that the plasma NfL level at any point during the disease course is a sensitive marker of disease activity and is associated with brain volume loss.

The main contribution of this study is that the strength of the association between the brain volume loss (MRI volumetry) seems to be higher than the association between pNfL and the "gold standard" measurement of current disease activity by NEDA-3. This is supported by results of the area under the ROC curve. We hypothetize that one of the advantages of $\mathrm{pNfL}$ is that unlike NEDA-3, pNfL also appears to cover false NEDA-3, as it appears to include spinal cord pathology and subclinical disease activity. On the other hand, it should be clarified in the future, whether pNfL reflects brain diseases other than MS (comorbidities) and the influence of age. However, because this is only an observational study, it is clearly necessary to verify the validity of our findings in the future in a wider range of SPMS and PPMS cohorts.

Neurofilaments have been extensively studied in relapsing-remitting multiple sclerosis ${ }^{1-6}$. Their role in progressive MS, where there is a particularly urgent need for sensitive valid biomarkers, is less clear. Several studies have shown that NfL levels in PMS are associated with current inflammatory activity ${ }^{22,24-26}$, current disability (EDSS, MSFC) (ref..$^{6,22,25,26}$ ), future disability worsening and future brain and spinal cord atrophy. A few studies have consistently shown NfL to be a marker of treatment response with immunosuppressive disease-modifying therapies $^{22,25,27}$. Our results support these findings, and moreover we conducted our study to determine the benefit of $\mathrm{pNfL}$ levels and BVL for our routine practice at a patient's regular annual evaluation.

While Kapoor et al. ${ }^{25}$ showed in the ASCEND study of natalizumab in SPMS that higher baseline blood NfL was associated with greater future 96-week brain atrophy, our study shows that NfL reflects brain atrophy of the past 12 months. This information can be useful in evaluation of treatment response.

Based on the results, we assume that pNfL has the potential to discriminate those patients with NEDA-3 and BVL, and therefore $\mathrm{pNfL}$ seems to be supportive biomark- 
er in making a treatment decision. We can recommend clinicians to include $\mathrm{pNfL}$ evaluation at a patient's regular annual monitoring measures of patients with progressive forms of multiple sclerosis as an auxiliary tool complementing the results of clinical and radiological follow-up. Based on strong association between pNfL levels, NEDA3 status and abnormal brain volume loss, unchanged NfL level compared to the previous one reflects no recent disease activity including neurodegenerative process. It can be useful for the treatment algorithm and identification of optimal treatment respondes.

There are several limitations of our study: our cohort is small and the follow-up was relatively short; a prospective study with a larger cohort of PMS patients could better explain NfL validity in individual PMS patient's disease course. We did not take into account all of the patients' comorbidities, vascular risk factors and aging, known factors which may affect NfL levels. Regarding brain volume measurement, there are several known obstacles in data interpretation or MRI volumetric techniques.

\section{CONCLUSION}

Plasma neurofilament levels, along with brain volume loss measurement, appear to be a useful tool for assessing current disease activity in patients with progressive MS.

\section{ABBREVIATIONS}

AR-BVL, Annual Rate Brain Volume Loss; BVL, Brain Volume Loss; CI, Confidence Interval; DMT, Disease Modifying Therapy; EDA, Evident Disease Activity; EDSS, Expanded Disability Status Scale; FLAIR, Fluidattenuated Inversion Recovery; GM, Gray Matter; MRI, Magnetic Resonance Imaging; MS, Multiple Sclerosis; MSFC, Multiple Sclerosis Functional Composite; NEDA, No Evident Disease Activity; pNfL, Plasma Neurofilament Light Chain; ROC, Receiver Operating Characteristics; PMS, Progressive Multiple Sclerosis; SIMOA, Single Molecule Array; WB, Whole brain.

Acknowledgements: We wish to thank to all patients who participated in the study. The study was supported by the Scientific Grant Agency of the Ministry of Education of the Slovak Republic (VEGA 1/0057/18 and VEGA 1/0540/20), the Slovak Research and Development Agency (under grant number APVV-15-0719), and research on Biomarkers of neurological disorders, UNLP and UPJŠ LF Košice. The funding bodies did not play any role in the collection, analysis and interpretation of the data, nor did they contribute to the writing of this manuscript.

Author contributions: All authors made substantial intellectual contributions to the study. JS, MV, MF: contributed to data collection; JS, MF: performed the initial literature review and wrote the first draft of the manuscript; JR, ZG, PM: supervised the work and performed statistical analyses; PU, JH, ES: performed and revised the laboratory tests; LF: analysed MRI data; JS, ZG, MV, JH, ES: contributed to revisions. All authors critically reviewed the manuscript and approved the final version of it.

Conflict of interest statement: The authors declare the following financial interests/personal relationships which may be considered as potential competing interests: JS has received personal compensation for consulting, serving on a scientific board, speaking or other activities, with received compensation for serving on a Scientific advisory board from Biogen, TEVA, Sanofi, Novartis, Roche and Merck, and for consultancy from Merck and Biogen. PM has no potential conflict of interest. JR has received personal compensation for consulting and speaking or other activities, with received compensation from Mundipharma. MF has received personal compensation for consulting, speaking or other activities from Biogen, Merck, Roche, Novartis, Sanofi and TEVA. PU has no potential conflict of interest. LF has no potential conflict of interest. MV has received personal compensation for consulting, serving on a scientific board, speaking or other activities, with received compensation for serving on scientific advisory board from Biogen, Merck, Novartis, Roche, Sanofi, and Teva. ZG has received personal compensation for consulting, serving on a scientific board, speaking or other activities, with received compensation for serving on scientific advisory board from Bayer, Biogen, Boehringer-Ingelheim, Merck, MSD, Novartis, Pfizer, Takeda and Teva. JH has no potential conflict of interest. ES has no potential conflict of interest.

Ethics approval and consent to participate: pproval for this study was provided by the Ethics Committee of the Louis Pasteur University Hospital in Košice (Approval number: 2017/EK/4005).

\section{REFERENCES}

1. Kuhle J, Barro C, Disanto G, Mathias A, Soneson Ch, Bonnier G, Yaldizi O, Regeniter A, Derfuss T, Canales M, Schluep M, Du Pasquier R, Krueger G, Granziera C. Serum neurofilament light chain in early relapsing remitting MS is increased and correlates with CSF levels and with MRI measures of disease severity. Mult Scler 2016;22(12):155059. doi: 10.1177/1352458515623365

2. Håkansson I, Tisell A, Cassel P, Blennow K, Zettenberg H, Lundberg P, Dahle C, Vrethem M, Ernerudh J. Neurofilament levels, disease activity and brain volume during follow-up in multiple sclerosis. J Neuroinflammation 2018;15:209. doi: 10.1186/s12974-018-1249-7

3. Kuhle J, Kropshofer H, Haering DA, Kundu U, Meinert R, Barro C, Dahlke F, Tomic D, Leppert D, Kappos L. Blood neurofilament light chain as a biomarker of MS disease activity and treatment response. Neurology 2019;92(10):e1007-e1015. doi: 10.1212/ WNL.0000000000007032

4. Siller N, Kuhle J, Muthuraman M, Barro Ch, Uphaus T, Groppa S, Kappos L, Zipp F, Bittner S. Serum neurofilament light chain is a biomarker of acute and chronic neuronal damage in early multiple scleosis. Mult Scler 2019;25(5):678-86. doi: 10.1177/1352458518765666

5. Novakova L, Zetterberg $H$, Sundström $P$, Axelsson $M$, Khademi M, Gunnarson M, Malmestrom C, Svenningsson A, Olsson T, Piehl F, Blennow K, Lycke J. Monitoring disease activity in multiple sclerosis using serum neurofilament light protein. Neurology 2017;89(22):2230-37. doi: 10.1212/WNL.0000000000004683

6. Disanto G, Barro C, Benkert P, Naegelin Y, Schadelin S, Giardiello A, Zecca C, Blennow K, Zettenberg H, Leppert D, Kappos L, Gobbi C, Kuhle J, Swiss multiple sclerosis cohort study group. Serum neuro- 
filament light chain: a biomarker of neuronal damage in multiple sclerosis. Ann Neurol 2017;81(6):857-70. doi: 10.1002/ana.24954

7. Gionvannoni G, Tomic D, Bright JR, Havrdova E. „No evident disease activity": The use of combined assessments in the management of patients with multiple sclerosis. Mult Scler 2017;23(9):1179-187. doi: $10.1177 / 1352458517703193$

8. Lublin FD, Reingold SC, Cohen JA, Cutter GR, Soelberg Sørensen $P_{t}$ Thompson AJ, Wolinsky JS, Balcer LJ, Banwell B, Barkhof F, Bebo B, Calabresi PA, Clanet M, Comi, G, Fox RJ, Freedman MS, Goodman AD, Inglese $\mathrm{M}$, Kappos L, Kieseier BC, Lincoln JA, Lubetzki C, Miller AE, Montalban X, O'Connor PW, Petkau J, Pozzilli C, Rudick RA, Sorman MP, Stüve O, Waubant E, Polman CH. Defining the clinical course of multiple sclerosis. Neurology 2014;83:278-86. doi: 10.1212/ WNL.0000000000000560

9. Radue EW, Barkhof F, Kappos L, Sprenger T, Haring DA, de Vera A, von Rosentiel P, Bright JR, Francis G, Cohen JA. Correlation between brain volume loss and clinical and MRI outcomes in multiple sclerosis. Neurology 2015;84(8):784-93. doi: 10.1212/WNL.0000000000001281

10. Sormani MP, Arnold DL, De Stefano N. Treatment effect on brain atrophy correlates with treatment effect on disability in multiple sclerosis. Ann Neurol 2014;75(1):43-49. doi: 10.1002/ana.24018

11. Sormani MP, Kappos L, Häring DA, Kropshofer H, Barro C, Leppert $D$, Tomic D, Kuhle J. Including blood neurofilament light chain in the NEDA concept in relapsing-remitting multiple sclerosis trials. Neurology (15 Supplement) S24.007 (2018) Corpus ID: 79895041 https://www.semanticscholar.org/paper

12. Weinstock-Guttman B, Medin J, Khan N, Korn JR, Lathi E, Silversteen J, Calkwood J, Silva D, Zivadinov R. MS-MRIUS Study Group. Assessing 'No Evidence of Disease Activity' status in patients with relapsingremitting multiple sclerosis receiving fingolimod in routine clinical practice: a retrospective analysis of the multiple sclerosis clinica and magnetic resonance imaging outcomes in the USA (MS-MRIUS) Study. CNS Drugs 2018;(329313):1-10. doi: 10.1007/s40263-0170482-4

13. Khalil $M$, Teunissen $C E$, Otto $M$, Piehl F, Sormani MP, Gattringer $T$, Barro C, Kappos L, Comabella M, Fazekas F, Petzold A, Blennow K, Zetterberg $\mathrm{H}$, Kuhle J. Neurofilaments as biomarkers in neurological disorders. Nat Rev Neurol 2018;14(10):577-89. doi: 10.1038/s41582 018-0058-Z

14. De Stefano N, Stromillo ML, Giorgio A, Bartolozzi ML, Battaglini M, Baldini M, Portaccio E, Amato MP, Sormani MP. Establishing pathological cut-offs of brain atrophy rates in multiple sclerosis. J Neuro Neurosurg Psychiatry 2016;87(1):93-99. doi: 10.1136/jnnp-2014309903

15. Uher T, Havrdova E, Sobisek L, Krasensky J, Vaneckova M, Seidl Z, Tyblova M, Ramasamy D, Zivadinov R, Horakova D. Is no evidence of disease activity an achieavable goal in MS patients on intramuscular interferon-beta-1a treatment over long-term follow-up? Mult Scler 2017;23(2):242-52. doi: 10.1177/1352458516650525

16. Kappos L, De Stefano N, Freedman MS, Cree BA, Radue EW, Sprenger T, Sormani MP, Smith T, Haring DA, Meier DP, Tomic D. Inclusion of brain volume loss in a revised measure of 'no evidence of disease activity' (NEDA-4) in relapsing-remitting multiple sclerosis. Mult Scler 2016:22(10):1297-305. doi: 10.1177/1352458515616701

17. Kurtzke RF. Rating neurologic impairment in multiple sclerosis: an expanded disability status scale (EDSS). Neurology 1983;33(11):1444452. doi: 10.1212/wnl.33.11.1444
18. Kuhle J, Barro C, Andreasson U, Derfuss T, Lindberg R, sandelius A, Liman V, Norgren N, Blennow K, Zettenberg H. Comparison of three annalytical platforms for quantification of of the neurofilament light chain in blood samples: ELISA, electrochemiluminiscence immunoassay and Simoa. Clin Chem Lab Med 2016;54(10):1655-661. doi: 10.1515/cclm-2015-1195

19. Steenwijk MD, Amiri H, Schoonheim MM, de Sitter A, Barkhof $F$, Pouwels PJW, Vrenken H. Agreement of MSmetrix with established methods for measuring cross-sectional and longitudinal brain atrophy. Neuroimage Clin 2017:5:843-53. doi: 10.1016/j.nicl.2017.06.034

20. Koch MW, Cutter GR, Giovannoni G, Uitdehaag BMJ, Wolinsky JS, Steinerman JR, Knappertz V. Comparative study of disability progression measures in PPMS. Analysis of the PROMiSe data set. Neuro Neuroimmuno Neuroinflamm 2017;4(4):e358. doi: 10.1212/ NXI.0000000000000358

21. European Medicines Agency Committee for medicinal products for human use. Guideline on clinical investigation of medical products for the treatment of multiple sclerosis EMA/CHPM/771815/2011, Rev.2, https://www.ema.europa.eu/en/documents/scientific-guideline/guideline-clinical-investigation-medicinal-products-treatmentmultiple-sclerosis (accessed 26 March 2015)

22. Kuhle J, Kropshofer H, Haring DA, Meinert R, Barro C, Dahlke F, Leppert D, Tomic D, Kappos L. Neurofilament light levels in the blood of patients with secondary progressive MS are higher than in primary progressive MS and may predict brain atrophy in both MS subtypes. Mult Scler J 2018;24:111.ECTRIMS Online Library.10/12/18; 232039; 286 https://onlinelibrary.ectrims-congress.eu/ectrims/2018/ ectrims-2018/232039

23. Sellebjerg F, Börnsen L, Ammitzbøll C, Nielsen JE, Vinther-Jensen T, Hjermind LE, von Essen M, Ratzer RL, Sorensen PS, Christensen JR. Defining active progressive multiple sclerosis. Mult Scler 2017;23(13):1727-35. doi: 10.1177/1352458517726592

24. Bar-Or A, Cross AH, Bennett JL, von Budingen HC, Carruthers $R$, Edwards $K$, Eggers $E$, Fallis R, Fiore D, Gelfand JM, Giacomini $P$, Greenberg B, Hafler DA, Longbrake EE, Assman B, lonete C, Kaunzner U, Lock C, Ma X, Musch B, Piehl F, Weber MS, Ziemssen T, Herman $A E$, Harp CT. Pretreatment cerebrospinal fluid (CSF) and serum neurofilament light (NfL) levels in patients with PPMS in the OBOE study are correlated and are higher in patients with PPMS with T1 Gd+ brain lesions. Mult Scler J 2019;25:494-95. ECTRIMS Online Library.09/12/19; 279308; P948 . https://onlinelibrary.ectrims-congress.eu/ectrims/2019/stockholm/279308

25. Kapoor R, Sellebjerg F, Hartung HP, Arnold DL, Freedman MS, Jeffery D, Miller A, Edwards KR, Singh CM, Chang I, Ren Z, Sangurdekar D, Zhu B, Sheikh S, Mehta D, Ho PR, Campbell N, Edwards M, Fisher E, Kieseier BC, Rudick RA, Plavina T. Natalizumab reduced serum levels of neurofilament light chain in secondary progressive multiple sclerosis patients from the phase 3 ASCEND study. Mult Scler J 24(2 Suppl):988. doi: $10.1177 / 1352458518799980$

26. Ferraro D, Guicciardi C, De Biasi S, Pinti M, Bedin R, Camera V, Vitetta F, Nasi M, Meletti S, Sola P. Plasma neurofilaments correlate with disability in progressive multiple sclerosis patients. Acta Neurol Scand 2020;141(1):16-21. doi: 10.1111/ane.13152

27. Barro C, Benkert P, Disanto G, Tsagkas C, Amann M, Naegelin $Y$, Leppert D, Gobbi C, Granziera C, Yaldizli Ö, Michalak Z, Wuerfel J, Kappos L, Parmar K, Kuhle J. Serum neurofilament as a predictor of disease worsening and brain and spinal cord atrophy in multiple sclerosis. Brain 2018;141:2382-91. doi: 10.1093/brain/awy154 